Conclusions The success of treatment for PA eradication is similar to those reported in cystic fibrosis and current treatment does not adversely impact on lung function.

\section{P243 DURATION AND CHOICE OF ANTIBIOTICS IN HOSPITAL ADMITTED COMMUNITY ACQUIRED PNEUMONIA PATIENTS OVER A PERIOD OF 5 YEARS IN NHS SOUTH EAST OF SCOTLAND}

doi:10.1136/thx.2010.151068.44

G C Choudhury, P M Mandal, A A Akram, A S Singanayagam, J C Chalmers, A H Hill. Royal Infirmary of Edinburgh, Edinburgh, UK

Introduction Clostridium difficile infection is associated with class of antibiotics as well as the duration of treatment. The aim of this study was to ascertain if the rate of hospital related Clostridium difficile varied with the duration and choice of antibiotics in those admitted to hospital with Community Acquired Pneumonia (CAP) over a period of 5 years (2005-2009).

Methods As a part of a prospective observational study of CAP in NHS Lothian, we investigated the duration and choice of antibiotics in hospital admitted CAP and the rate of Clostridium difficile infection in these patients over a period of 5 years (2005-2009). For multiple comparisons, we used the KruskalWallis test for numerical data and Chi squared test for the categorical data.

Results The duration and choice of antibiotics are tabulated in the Abstract P243 Table 1. The length of antibiotics used for severe CAP was therefore, longer than mild CAP. The duration of antibiotics for all severity of CAP, has not changed between 2005 and 2009. There has been a reducing usage of cephalosporins and macrolides with rising use of co-amoxiclav. The contribution of CAP as a cause for Clostridium difficile, however, remains unchanged.

Conclusion In conclusion our study shows that the proportion of Clostridium difficile cases due to CAP has not changed in NHS Lothian between 2005 and 2009, despite significant reduction of cephalosporins and macrolide use. Randomised controlled trials are needed to assess whether reducing the length of treatment will influence Clostridium difficile rates.

\section{P244 ADHERENCE WITH DEPARTMENT OF HEALTH GUIDELINES FOR THE MANAGEMENT OF PANDEMIC H1N1 INFLUENZA IN SECONDARY CARE}

doi:10.1136/thx.2010.151068.45

M M Khan, R B Gore, S J Fowler. Lancashire Teaching Hospitals NHS Foundation Trust, Preston, UK

Background Prior to the 2009 swine flu pandemic, there was uncertainty about the expected incidence, morbidity and mortality from the disease. The Department of Health (DoH) compiled guidelines for assessment and management of children and adults admitted to hospital with suspected H1N1 infection.

Objectives We evaluated whether adults with suspected H1N1 infection were appropriately diagnosed, investigated and managed within an NHS Foundation Trust during a period of maximal incidence when $\mathrm{DoH}$ guidance was accessible, with the aim of improving care for patients during future pandemic flu outbreaks and utilising hospital resources efficiently.

Methods Patient notes of suspected cases of adult H1N1 infection between July and December 2009 were retrospectively reviewed to identify how many met the diagnostic criteria, underwent relevant investigations and were prescribed a neuraminidase inhibitor as compared with DoH guidelines. The relationship between the initial consultant's diagnosis and the final diagnosis was also considered.

Results Seventy cases of suspected swine flu were identified, and full documentation was available for review in 61 of these. All patients were tested for swine flu and overall $26 \%$ of patients were H1N1 positive, including $6 \%$ of those patients who did not fulfil the diagnostic criteria. Of patients clinically suspected of having swine flu $34(56 \%)$ did not fulfil the diagnostic criteria, although two of those. were found to be H1N1 positive. Minimum recommended investigations were performed as follows; routine bloods and chest $x$-ray in $85 \%$; blood cultures in $33 \%$; sputum cultures in $15 \%$; and urinary pneumococcal antigen testing in $3 \%$. Antiviral medication was not prescribed in $31 \%$ of patients suspected of having swine flu. In $74 \%$ of the cases, the initial consultant's diagnosis matched the final diagnosis; this was true for $70 \%$ of the patients who were H1N1 positive.

Conclusions Increased awareness of the available guidelines is required to optimise diagnosis and management, and minimise the likelihood of potentially unsafe, incorrect diagnoses. This requires education of healthcare staff of available guidance, and further audit following the next outbreak of pandemic flu with the aim of safely and efficiently guiding clinical practice.

\section{P245 ASSESSMENT OF ACUTE ILLNESS SEVERITY AND RADIOLOGICAL EXTENT IDENTIFY PATIENTS AT HEIGHTENED RISK OF DEVELOPING MAJOR PNEUMONIC PROGRESSION IN INFLUENZA A H1N1/2009 INFECTION}

doi:10.1136/thx.2010.151068.46

J Choy, A Draper, E Ribbons, I Vlahos, S Grubnic, T Bicanic, P Riley, M WansbroughJones, F Chua. St George's Healthcare NHS Trust, London, UK

Introduction In 2009, high transmissibility of a novel influenza A H1N1 virus produced a global outbreak of febrile pneumonic illness. Clinical criteria for its' diagnosis suffered from low sensitivity and specificity. We evaluated clinical, laboratory and radiological abnormalities in virologically proven H1N1/2009 infection to identify risk factors associated with severe pulmonary involvement.

Abstract P243 Table 1 Change in components of the NHP and LCO

\begin{tabular}{|c|c|c|c|c|c|c|c|c|}
\hline \multirow[b]{2}{*}{ Year } & \multicolumn{3}{|c|}{ Duration of antibiotics (days) Mean \pm SD } & \multirow{2}{*}{$\begin{array}{l}\text { p-Value } \\
\text { (year wise) }\end{array}$} & \multicolumn{3}{|l|}{ Choice of antibiotics } & \multirow{2}{*}{$\begin{array}{l}\text { Proportion of } C \text {. difficile } \\
\text { cases due to CAP (\%) }\end{array}$} \\
\hline & Mild CAP & Moderate CAP & Severe CAP & & Cephalosprins (\%) & Macrolides (\%) & Coamoxiclav & \\
\hline 2005 & $7.89 \pm 3.68$ & $9.61 \pm 4.32$ & $9.71 \pm 2.94$ & $<0.0001$ & 24.8 & 65.9 & 50.8 & 10.4 \\
\hline 2006 & $8.33 \pm 2.37$ & $8.55 \pm 2.06$ & $10.57 \pm 3.36$ & $<0.0001$ & 29.2 & 75.5 & 35.9 & 9.2 \\
\hline 2007 & $8.67 \pm 3.46$ & $9.51 \pm 4.3$ & $9.87 \pm 3.01$ & $<0.0001$ & 19.7 & 74 & 61.1 & 9.9 \\
\hline 2008 & $9.08 \pm 4.05$ & $9.79 \pm 4.43$ & $9.93 \pm 3.00$ & $<0.0001$ & 15.1 & 61.8 & 59.2 & 10.8 \\
\hline 2009 & $8.24 \pm 3.66$ & $10.26 \pm 5.83$ & $9.78 \pm 3.51$ & $<0.0001$ & 7.9 & 54.6 & 71.3 & 8.9 \\
\hline $\begin{array}{l}\mathrm{p} \text {-Value (in each } \\
\text { subgroup of CAP) }\end{array}$ & 0.1 & 0.06 & 0.2 & $\begin{array}{l}\text { p-Value (in each } \\
\text { antibiotic subgroup) }\end{array}$ & $<0.0001$ & $<0.0001$ & $<0.0001$ & \\
\hline
\end{tabular}


Methods Data were collected at one London acute care Trust during two phases (June-August and September-November 2009) of the pandemic. Initial 24-h Acute Physiology and Chronic Health Evaluation (APACHE)-II scores and radiological findings were evaluated. All data were analysed by logistic regression (STATA)

Results 64 patients (31 female, 33 male; median age 32) hospitalised with H1N1/2009 were studied, comprising 22 white British, 19 African-Caribbean, 21 Asian and 2 of other ethnicity. Only three patients were aged $>60$ and only two deaths were encountered. Six pregnant patients were treated, including five in phase 2. Higher mean APACHE-II scores (7.9 vs 6.6) were recorded during this phase but were unrelated to demographic factors. ICU admission rate was high (12/64 patients, 19\%; including three who were pregnant). Asthma was the commonest co-morbidity (17/64 patients, 27\%) but only featured in two ICU cases. By contrast, pregnancy was a predictor of ICU admission (Pearson $\chi^{2} 4.2, \mathrm{P}$ $0.03)$. Asian males more than any other demographic were also more frequently identified than expected for this population $\left(\chi^{2}\right.$ 4.94, $\mathrm{p}=0.026$ ). Radiographic abnormalities were noted in $\geq 2$ quadrants of 28 (44\%) initial chest films, most frequently basal peribronchial thickening and perihilar opacities. Progressive infiltrates or multifocal consolidation were noted in 15/58 (26\%) patients, two-thirds $(9 / 15)$ of whom ultimately requiring intubation. Nosocomial infections or co-pathogens were found in six cases, all with underlying malignancy, transplanted organ or pregnancy.

Conclusions Most patients hospitalised with H1N1/2009 were young, with mild clinical and radiological disease. Asian males were more likely to be hospitalised with the illness, while pregnancy was correlated with an increased likelihood of ICU admission. Neither subgroup had evidence of worse acute illness severity. Basal nonlobar changes were the commonest radiographic finding. Overall, diffuse early abnormalities frequently preceded the development of severe and progressive pneumonia.

\section{P246 SUSPICION-PROMPTED TESTING REVEALS MISSED OPPORTUNITIES FOR DIAGNOSING NEW HIV CASES AMONG PATIENTS WITH COMMUNITY-ACQUIRED PNEUMONIA}

doi:10.1136/thx.2010.151068.47

S Safavi, F J K Chua, P Reilly, G K Russell. St Georges Hospital, London, UK

Introduction National UK policy for HIV testing ${ }^{1}$ recommends offering an HIV test to all patients with HIV indicator diseases such as tuberculosis (TB), Pneumocystis pneumonia, aspergillosis and bacterial pneumonia. Universal testing is advocated for all adults registering with a GP and all general medical hospital admissions in regions where HIV prevalence is $>2 / 1000$ population. Although 'opt out' HIV testing is a standard practice for patients with $\mathrm{TB}$, testing falls far short in other clinical settings.

Method A large number of community-acquired pneumonia (CAP) cases across three south London boroughs where HIV prevalence exceeds $2 / 1000^{2}$ are managed at our institution. All cases of CAP in 2008 (with one or more of: radiographic consolidation, CAP clinical case definition fulfilment, positive microbiology or pneumococcal urinary antigenaemia) were included in the present analysis. Demographic, HIV sampling and outcome data were collected.

Results 618 patients ( 325 or $53 \%$ male, 293 female) with a diagnosis of CAP were identified. The majority $(500,81 \%)$ were aged $>50$. Of the total, only 23 (3.7\%) patients underwent HIV testing, of whom 1 in 4 $(26 \%)$ tested positive. Half represented new diagnoses while the remainder had prior documentation of HIV positivity. Overall, 9/60 $(15 \%)$ of ethnically black (African-Caribbean) patients were tested, compared to only 6/429 (1.4\%) of White British patients. The rate of testing varied between age groups: $16 \%(19 / 118)$ of those aged $15-50$ were tested, in contrast to only $0.8 \%(4 / 500)$ of patients aged over 50 . Information regarding patients were declined testing was not available. Discussion Of individuals diagnosed with CAP who were HIVtested, the proportion of 'incident' positive testing (13\%) is approximately 5 times higher than expected if a policy of universal testing was practised. HIV testing was more often performed on younger or black patients. Despite serving a high prevalence population, HIV testing at this and likely many other institutions is under-utilised by medical teams even for patients with an HIV indicator pathology. Management of CAP in secondary care represents an opportunity to prevent a delayed diagnosis of HIV. Barriers to HIV testing need to be identified to reduce HIV mortality associated with late diagnosis.

\section{REFERENCES}

1. UK National Guidelines for HIV Testing (http://www.bhiva.org).

2. HIV in the United Kingdom: 2009 report. Health Protection Agency published November 2009

\section{P247 HIGH LEVELS OF IRON IN THE SOIL ARE ASSOCIATED WITH AN INCREASED INCIDENCE OF BOVINE TUBERCULOSIS IN CATTLE}

doi:10.1136/thx.2010.151068.48

G V O'Donovan, H J Milburn. Department of Respiratory Medicine, Guy's and St Thomas' NHS Foundation Trust, London, UK

Background Numbers and proportion of cases of bovine tuberculosis (BTB) in cattle are increasing, largely in the west country and Wales with few if any cases in the south and north east. Work by DEFRA demonstrates that TB in cattle is mutifactorial, as in humans, making some herds at greater risk of $\mathrm{TB}$ than others. Micronutrients could be one of these factors. Deficiencies, but also excesses, may cause secondary immunodeficiency and infection related morbidity in man. Different soil types contain different quantities of these trace elements and this will be reflected in pasture, hay and silage.

Objectives To determine whether certain rock and soil types predominate in areas with high incidence of BTB, and whether there is a case for investigating micronutrients in cattle.

Methods Information on cases of BTB and latest mapping of cases was provided by the Veterinary Research Laboratories (UK). Using information from the British Geological Survey and geological mapping, rock and soil types and their mineral contents were compared with density of cases of BTB.

Results There is a significant difference in the proportion of confirmed new incidents of BTB in the west of England $(6.8 / 100$ herds) and all other areas $(\mathrm{p}<0.001)$, and Wales $(3.2 / 100)$ and the north $(0.8 / 100)$ and east $(0.3 / 100)$ of England $(p<0.001)$. The rock types present in the west of England and Wales are rich in iron and aluminium while those in areas largely free of BTB are rich in calcium salts and oxides of silicon. Soils reflect the mineral content of the underlying rocks.

Discussion Iron deposits are common in areas with high numbers of cases of BTB, affecting quantities in pasture, hay, silage, and earthworms, a staple component of the diet of badgers, who are frequently blamed for this disease. Excess iron is associated with increased susceptibility to TB and more aggressive disease in man, and mycobacteria responsible for BTB need iron for their survival within the host.

Conclusions There is an association between BTB and high levels of iron in the soil. Further work is needed to determine levels of iron in affected cattle and its effect on immune responses. 